\title{
Numerical Simulation of Hot Spot Growth in Detonation with Regard to the Turbulent Mechanism of Energy
}

\section{Transfer}

Yury Vasilyevich Yanilkin ${ }^{1 *}$, Vitaly Grigoryevich Morozov ${ }^{1}$, Irina Igorevna Karpenko and Olga Nikolaevna Chernyshova ${ }^{1}$

1. Russian Federal Nuclear Centre, VNIIEF, Sarov 607200, Russia

\begin{abstract}
The paper presents the results of numerical modeling of hot spot growth process in detonation with account for turbulent mixing. The performed investigation has shown that large-scale HE (High explosives) particles mix up and split down to smaller sizes in the result of shock wave impact, instability development on the HE-EP (Explosion product) interface and vortex flow; at these sizes, due to the developed surface of the HE-EP contact, HE has enough time to get heated (energy transfer from EP), and the decomposition reaction effectively continues. Numerical modeling make the calculation of the hot spot growth rate (about 100-200 $\mathrm{m} / \mathrm{s}$ ) possible. This has proved the hypothesis saying that at mechanical material transport the turbulence in the reaction zone plays an important role and it must be taken into account in the detonation theory.
\end{abstract}

Key words: Hot spot, detonation, turbulent mixing, numerical simulation.

\section{Nomenclature}

If not additionally specified, the following notations and units are used in this paper:

$\mathrm{t} \quad$ Time $[\mathrm{t}]=\mu \mathrm{s}$

L Length $[\mathrm{L}]=\mathrm{cm}$

$\mathrm{u}, \mathrm{c}_{0}$ Mass and sound velocities $[\mathrm{u}],\left[\mathrm{c}_{0}\right]=\mathrm{km} / \mathrm{s}$

$\rho$ Density $[\rho]=\mathrm{g} / \mathrm{cm}^{3}$

$\mathrm{Q}$ Caloricity $[\mathrm{Q}]=\mathrm{kJ} / \mathrm{g}$

$\mathrm{C}_{\mathrm{v}} \quad$ Heat capacity $\left[\mathrm{C}_{\mathrm{v}}\right]=\frac{\mathrm{kJ}}{\mathrm{g} \cdot{ }^{\circ} \mathrm{K}}$

$\chi \quad$ Coefficient of thermal conductivity $[\chi]=\frac{\mathrm{J}}{\mathrm{m} \cdot \mathrm{s} \cdot{ }^{\circ} \mathrm{K}}$

$\mathrm{T}$ Temperature $[\mathrm{T}]={ }^{0} \mathrm{~K}$

P Pressure $[\mathrm{p}]=10^{-4} \mathrm{GPa}$

e Specific energy $[\mathrm{e}]=\mathrm{kJ} / \mathrm{g}$

$\mathrm{Z} \quad$ Arrhenius kinetics parameter $[\mathrm{Z}]=1 / \mathrm{s}$

\section{Introduction}

Solid high explosives are heterogeneous. They have cavities and cracks, grain boundaries and interphase boundaries. Structural defects (cavities, interphase and

"Corresponding author: Yury Vasilyevich Yanilkin, professor, research field: computational mathematics. E-mail: n.yanilkina@mail.ru. intergrain boundaries, lattice defects, cracks) influence the shock-wave sensitivity and dynamics of shock wave transition to detonation. HE (High explosive) initiation also takes place when the SW (Shock wave) energy is not sufficient for homogeneous HE heating up to the flash bang temperature. Localization of shock wave energy on the structural defects results in local heating and initiation of disintegration reaction in so called "HS (Hot spots)".

The hot spot concept and the spot-based mechanism of detonation initiation and development are the basic conception of the modern physics of explosion [1-6]. In the frames of this theory many phenomena are naturally explained by the fact that the shock wave contact with structural defects results in the localization of energy dissipation at detached spots.

For convenience the initiation pattern for heterogeneous HE can be divided into three stages.

Hot spot forming: This is the stage from the moment of HE compression with shock wave to the start of chemical reaction in local spots. This stage 
continues for about $1 \mathrm{~ns}$ and consists of the generation of localized heated spots in the vicinity of cavities and boundaries of the grains due to the effects of heated gas in the cavities, microcumulative effects when the cavities collapse, inter-grain friction and plastic deformations [3-6].

Hot spot growth (or dying out) depending on the pressure in the surrounding "cold" HE prior to the contact with neighboring spots. At this phase the disintegration reaction in the surrounding $\mathrm{HE}$ develops. The characteristic time of this process is much longer and determines the time of detonation inductance of $\sim 1 \mu \mathrm{s}$. If average $\mathrm{HE}$ grain size is about $10 \div 100 \mu \mathrm{m}$, than the spot combustion boundary velocity with account for the inductance time should be about $100 \mathrm{~m} / \mathrm{s}$. The velocity of regular laminar spot burning is less than $1 \mathrm{~m} / \mathrm{s}$. Papers [7, 8] consider the problem about the HS growth in HE basing on TATB in 1D approximation and the obtained HS growth velocity is less than $1 \mathrm{~m} / \mathrm{s}$. This indicates the necessity of more detailed analysis of physical processes that determine the hot spot growth rate in shock-compressed HE.

Inter-spot interaction and merging: At this phase the quick high-temperature and high-pressure reaction completion takes place at the moment when the interacting areas start merging, which is the process that supports fast transition to self-sustained stationary detonation process (the explosion proper).

The key stage of the detonation development is the process of hot spot growth resulting in the shock wave transition into detonation, and its dependence on pressure is determined by the energy transfer process. We can suppose that in a large-scale HE burning the dependence of the burning front velocity on pressure is also determined, firstly, by the energy transfer process. Various theories of this process exist, and the dominant one is based on the molecular thermal conductivity. However, the estimations of the growth rate for HS are less than $100 \mathrm{~m} / \mathrm{s}$. Such HS growth rate can be obtained if the coefficient of thermal conductivity for EP exceeds its value in normal conditions by 3 or more orders of magnitude, which is physically incorrect.

In this paper the turbulent mixing is considered as such mechanism, which possibility has been expressed before [9-11]. Due to high intensity of turbulent mixing the turbulent flows have increased ability of heat transfer and accelerated propagation of chemical reactions. However, the mentioned papers interpreted the turbulence as a small-scale process and neglected its effect on the burning process. The first 2D numerical study that affirmed the turbulent mechanism of energy transfer has been described by the authors of this paper in [12].

We believe that the general physical picture of the process is as follows. Turbulence occurs due to the multi-dimensional character of the burning process and HS growth, as well as because of gas dynamic instability on the burning front. It mixes up large-scale HE particles and at that splits them down to smaller sizes, at which due to the developed surface of the HE-EP contact, HE has enough time to get heated (energy transfer from EP to $\mathrm{HE}$ ), and the decomposition reaction effectively continues.

This paper is the continuation of the numerical study presented in [12]. In this paper HE burning modeling was made in the $3 \mathrm{D}$ approximation using the FCD code EGAK [13] without any turbulence models, which means it was DNS modeling. For the reader's convenience we also provide the results of paper [12], where the 2D EGAK simulations using the к- $\varepsilon$ model are shown.

\section{2D Simulations}

\subsection{Simulation Setting}

Initial geometry for 2D axially symmetrical simulations is shown in Fig. 1.

A square with the side $5 \times 10^{-3}$ is considered. The quarter sphere with the radius $\mathrm{R}=10^{-3}$ is occupied by heated EP in accordance with the HE caloricity and thermal capacity. The rest area is occupied by cold HE. 


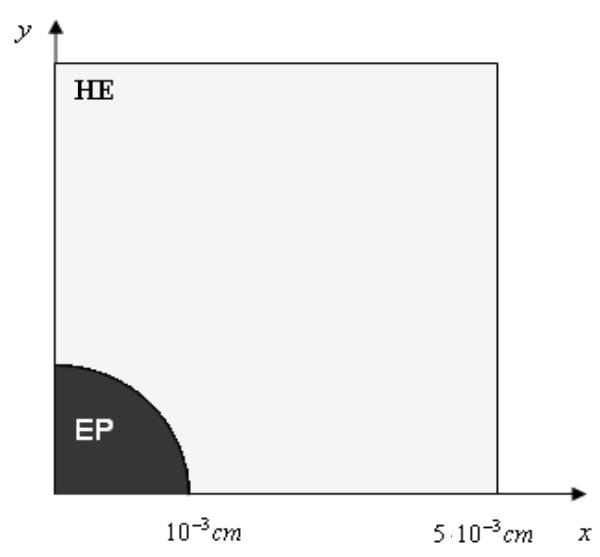

Fig. 1 Initial geometry of 2D problem.

Thereby, the EP emergence and heating processes are not modeled here.

The equation of HE burning kinetics has the following form (Arrhenius law):

$$
\frac{\mathrm{dW}}{\mathrm{dt}}=-\mathrm{Z} \cdot \exp \left(-\frac{\mathrm{T}_{\mathrm{a}}}{\mathrm{T}}\right) \cdot \mathrm{W}
$$

Here $\mathrm{W}$ is the HE mass concentration, $\mathrm{T}$ is the HE temperature, activation temperature is $\mathrm{T}_{\mathrm{a}}=30,000, \mathrm{Z}$ $=5 \times 10^{13}$.

The Eqs. of state of the Mie-Grueneisen type were used for HE:

$$
\begin{aligned}
& \mathrm{P}=\mathrm{P}_{1}+\mathrm{P}_{2} \\
& \mathrm{E}=\mathrm{E}_{1}+\mathrm{E}_{2}
\end{aligned}
$$

where,

$$
\begin{gathered}
\mathrm{P}_{1}(\delta)=\frac{\mathrm{C}_{0}^{2} \cdot \rho_{0}}{\mathrm{n}} \cdot\left(\delta^{\mathrm{n}}-1\right) \\
\mathrm{E}(\delta)=\frac{\mathrm{C}_{0}^{2}}{\mathrm{n}} \cdot\left(\frac{\delta^{\mathrm{n}-1}}{\mathrm{n}-1}+\frac{1}{\delta}-1\right) \\
\mathrm{E}_{1}(\delta)=\frac{1}{\rho_{0}} \cdot \int_{1}^{\delta} \frac{\mathrm{P}_{1}(\tau)}{\tau^{2}} \mathrm{~d} \tau \\
\mathrm{P}_{2}=\gamma \cdot \rho_{0} \cdot \delta \cdot \mathrm{E}_{2}
\end{gathered}
$$

Here $\delta=\rho / \rho_{0}$ is relative compression, The following $\mathrm{EoS}$ parameters were used in the simulations: $\mathrm{C}_{\mathrm{V}}=0.00135, \mathrm{C}_{0}=2.43, \mathrm{n}=6.2, \gamma=2.1$, $\rho_{0}=1.93, Q=3.65$.

The Zubarev EoS [14] was used for EP with the following parameters: $\mathrm{C}_{\mathrm{V}}=0.00102 ; \mathrm{A}=2.2155 ; \mathrm{F}=$ $75.802 ; \mathrm{n}=0.6 ; \xi=4.383 ; \gamma_{0}=0.5$.

In the simulations, apart from gas dynamics and kinetics, various combinations of thermal conductivity and turbulence under the $\kappa-\varepsilon$ model were included into the calculations. Variations of physical processes in the simulations are given in Table 1 . The main $2 \mathrm{D}$ simulations were performed on the uniform Eulerian grid $100 \times 100$. For the purposes of results convergence estimation, additional two simulations were performed on the $50 \times 50$ and $150 \times 150$ grids with simultaneous accounting for all processes specified above. The settings of all simulations are showed in Table 1.

Note, that in the EGAK code the heat conduction equation is solved in terms of mean energy in a cell. After the new mean energies are found, the energy in mixed cells is distributed between the materials basing on some kind of supposition. In this simulations we applied the supposition about instantaneous balancing of components' temperature. For the materials mixed up due to turbulence this supposition is deemed to be quite appropriate. In fact this means, that in such cell

\begin{tabular}{|c|c|c|c|c|c|}
\hline Run & Grid & Gas dynamics & Kinetics & Heat transfer & Turbulence $(\kappa-\varepsilon)$ \\
\hline 1 & $100^{2}$ & + & + & - & - \\
\hline 2 & $100^{2}$ & + & + & + & - \\
\hline 3 & $100^{2}$ & + & + & - & + \\
\hline 4 & $100^{2}$ & + & + & + & + \\
\hline 5 & $50^{2}$ & + & + & + & + \\
\hline 6 & $150^{2}$ & + & + & + & + \\
\hline
\end{tabular}
the heat exchange between materials is realized instantaneously, which conforms to the supposition about homogeneous character of materials mixing in a cell.

Table 1 Various combinations of physical processes taken into account in 2D simulations (“+” means the presence of process, “-” means the absence of process). 


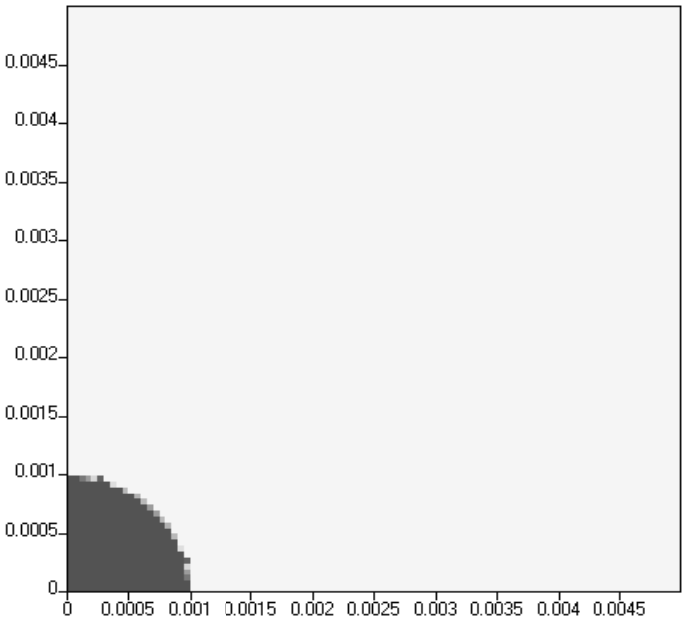

(a)

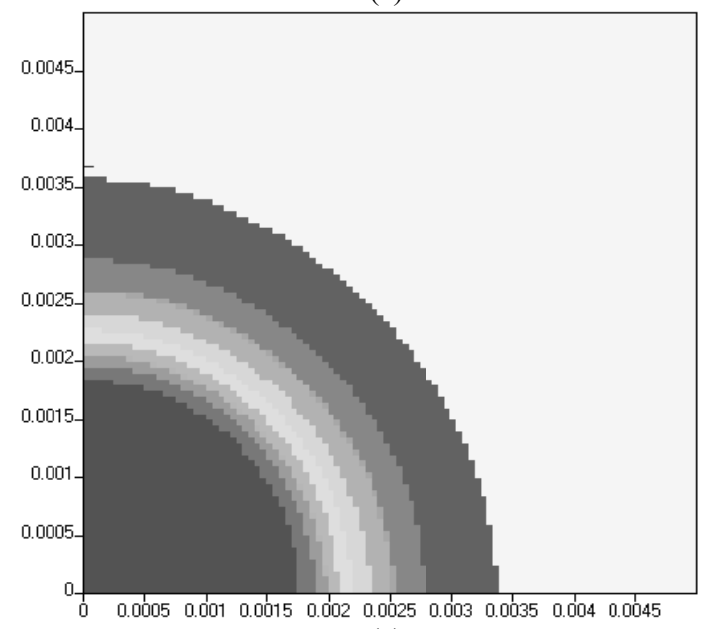

(c)

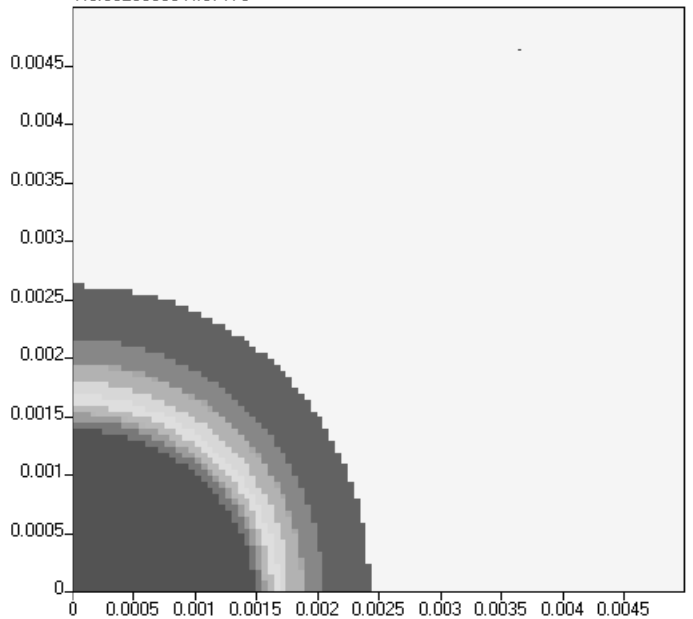

(b)

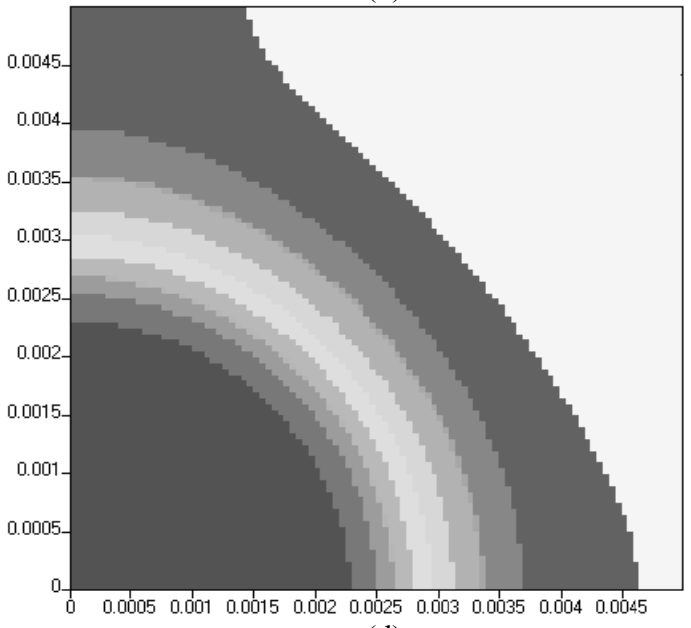

(d)

Fig. 2 EP concentration in the 2D simulation $4:(a) t=0$, (b) $t=0.002$, (c) $t=0.005$ and $(d) t=0.008$.

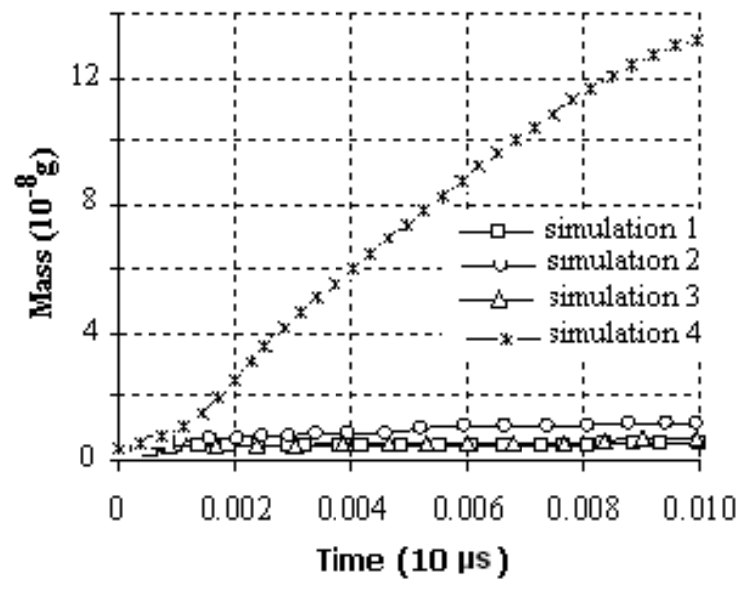

Fig. 3 Time dependence of EP mass in 2D simulations.

\subsection{Simulation Results}

Fig. 2 shows the EP concentrations at several times from the simulation 4 (with account for turbulence and thermal conductivity). They demonstrate the process of the hot spot growth.

Fig. 3 shows the time dependences of EP mass for four simulations (1-4) with account for various combinations of thermal conductivity and turbulence. The obtained result is as follows: simultaneous inclusion of turbulence and thermal conductivity gives physically reasonable growth rate of hot spot and in the simulation transmits the process characteristic physical features. At the same time individual accounting for each process (thermal conductivity or turbulence) does not give the required hot spot growth rate.

Fig. 4 shows the time dependence of the EP radius in the simulation 4 with account for all processes. We can see, that the curve rather quickly reaches the linear 


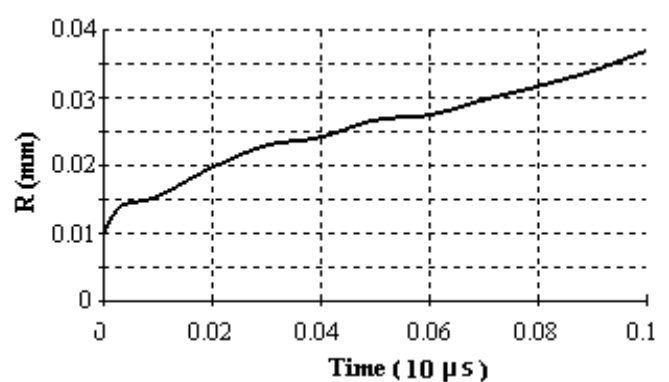

Fig. 4 Time dependence of the EP radius in the simulation 4.

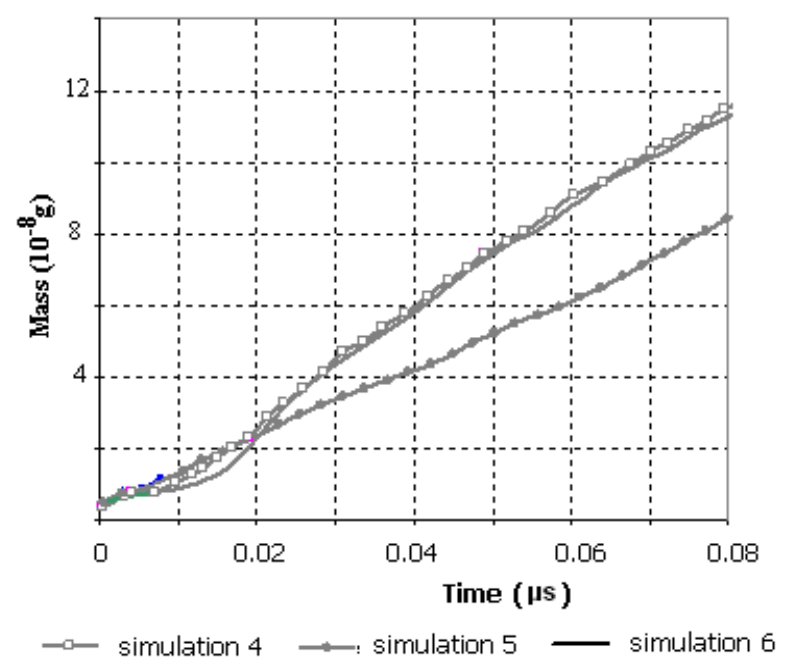

Fig. 5 Time dependences of EP mass in 2D simulations with account for thermal conductivity and turbulence on varied computational grid.

growth law where the mean HS growth rate is: $\Delta \mathrm{R} / \Delta \mathrm{t} \sim 200 \mathrm{~m} / \mathrm{s}$.

The graphs in Fig. 5 show the time dependences of the EP mass in the simulations performed on various grids. We can see, there is convergence at the grid refinement. At that the results of simulations 4 and 6 utterly match each other.

\section{3D Simulations}

3D simulations were performed on fixed computational grids in Eulerian variables with $\mathrm{HE}$ and EP separation into different materials using the concentrations method (volume of fluid). Turbulence models were not used in 3D simulations: from the point of view of turbulence such simulations were direct numerical modeling. All 3D simulations were performed with account for the process of heat conductance. Note, that in these simulations, as well as above, the supposition about the temperature equity for components in mixed cells is used. In fact this means, that in such cells the heat exchange between materials is realized instantaneously, which conforms to the supposition about homogeneous character of materials mixing in a cell.

In contrast to the preceding section, the HE structure configuration with gas inclusion was taken into account in 3D simulations. Since the real configuration could be difficult to represent, two elementary ones were chosen: a sphere and a tetrahedron.

\subsection{Simulation of Hot Spot Formation around Heated Gas Inclusion}

In this simulation series we simulated the hot spot formation from EP after shock way passing through $\mathrm{HE}$ and spherical gas inclusion. The process of shock wave passing is not simulated in this series; it is supposed that at the initial moment of time the gas inclusion is heated with shock wave.

\subsubsection{Simulation Setting}

Initial geometry is a cube with the side of $5 \times 10^{-3}$. The spherical octant with the radius $\mathrm{R}=10^{-3}$ is occupied by the gas with the input parameters $\rho_{0}=0.4$, $\mathrm{p}_{0}=0.32, \mathrm{e}_{0}=4$. EoS-"ideal gas" with the following parameters: $\gamma=1.2, \quad C_{V}=0.0004$. Thus, the gas temperature at the initial moment is $\mathrm{T}=\mathrm{e} / \mathrm{C}_{\mathrm{V}}=10,000$. HE with the initial parameters $\left(\rho_{1}=1.91, \mathrm{p}_{1}=8, \mathrm{e}_{1}=\right.$ 2.02349) occupy the rest area. The Eqs. of gas state, $\mathrm{HE}$ and EP, as well as the Arrhenius kinetics parameters are the same as in the main simulations of Section 1.

The used coefficients of thermal conductivity were: $\chi_{\text {gas }}=150, \chi_{\mathrm{BB}}=0.5016, \chi_{\mathrm{EP}}=1$, however, in one of the simulations these coefficients were increased by the order of magnitude for all materials.

The main simulations were performed on the homogeneous grid $100 \times 100 \times 100$. Apart from that, one simulation was made on the grid $400 \times 400 \times 400$. Table 2 shows the variants of the first series simulations 
Table 2 Variants of the first series 3D simulations.

\begin{tabular}{lllll}
\hline Run & Cell size & $\mathrm{Z}$ & Gas inclusion shape & Coefficients of thermal conductivity \\
\hline 1 & 0.5 & $5 \times 10^{13}$ & Sphere & $\chi_{\text {gas }}=150 ; \chi_{\mathrm{HE}}=0.5016 ; \chi_{\mathrm{EP}}=1$ \\
2 & 0.5 & $5 \times 10^{14}$ & Sphere & $\chi_{\text {gas }}=150 ; \chi_{\mathrm{HE}}=0.5016 ; \chi_{\mathrm{EP}}=1$ \\
3 & 0.5 & $5 \times 10^{13}$ & Sphere & $\chi_{\text {gas }}=1,500 ; \chi_{\mathrm{HE}}=5.016 ; \chi_{\mathrm{EP}}=10$ \\
4 & 0.125 & $5 \times 10^{13}$ & Sphere & $\chi_{\text {gas }}=150 ; \chi_{\mathrm{HE}}=0.5016 ; \chi_{\mathrm{EP}}=1$ \\
5 & 0.125 & $5 \times 10^{13}$ & Tetrahedron & $\chi_{\text {gas }}=150 ; \chi_{\mathrm{HE}}=0.5016 ; \chi_{\mathrm{EP}}=1$ \\
\hline
\end{tabular}

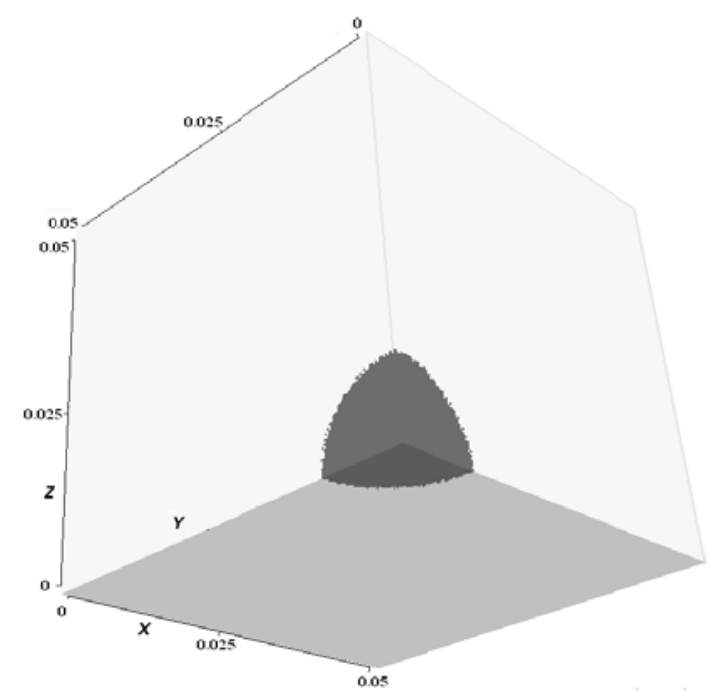

(a)

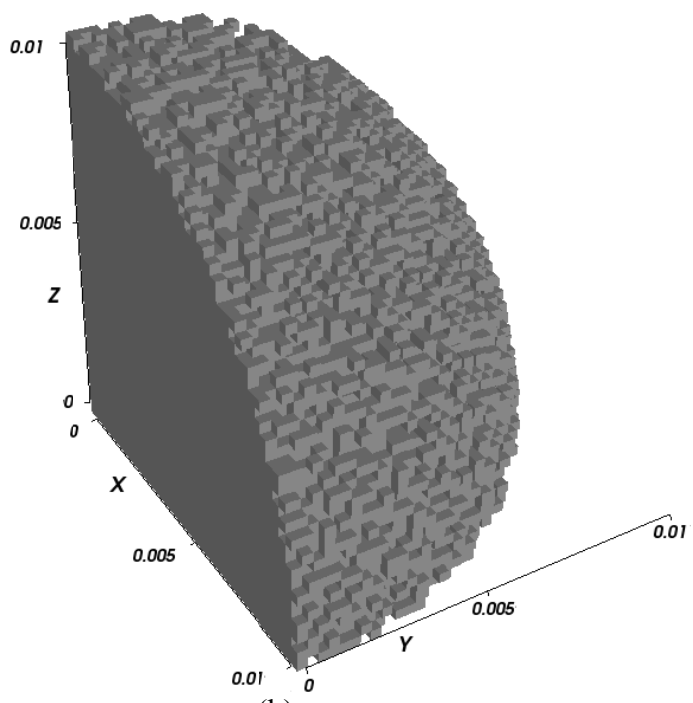

(b)

Fig. 6 Initial geometry of 3D problem: (a) - full geometry and (b) - gas inclusion.

with varied amount of cells, coefficients of thermal conductivity and kinetic parameter Z. The "rigid wall" boundary condition was set on the external boundaries of the cube.

Initial perturbations are set on the gas-HE interface for the turbulence development: we take a cube with a two-cell side and in a random way introduce into it either gas, or HE. The initial gas shape is showed in Fig. 6. Note, that in the simulation on a more refined grid the amplitudes of the initial perturbations are smaller in accordance to the cell sizes.

It is worth mentioning, that the simulation parameters were taken in accordance with the analytical calculations from $[15,16]$. The variations of the thermal conductivity parameters in the simulations are associated with the absence of reliable data on the coefficients of thermal conductivity for the materials, especially HE, under shock compression. As for the Arrhenius kinetics and its parameters, in these simulations it was used as an indicator or threshold "breaker" of the disintegration reaction at certain $\mathrm{HE}$ heating, and its parameters were chosen in such way that at the given EoS's the critical regime of a unit spot growth was realized on the background of the non-reacting $\mathrm{HE}$. In the simulation we checked that the increase by the order of magnitude of the pre-exponent $\mathrm{Z}$ value in the Arrhenius kinetics resulted in the homogeneous chemical reaction in the volume, which was consistent with the physical logics.

The real shape of gas inclusions formed on the juncture point of several HE grains is not spherical [17] (Fig. 7), therefore the simulation 5 (Table 2) with the gas inclusion as a tetrahedron was performed. At that the gas inclusion is located inside the cubic computational domain to reduce the effect of rigid walls on the process development, which required the increase of the cube size up to $6 \times 10^{-3}$. Otherwise the simulation setting matches the setting of the simulation 4 for refined grid $\left(\mathrm{h}=1.25 \times 10^{-5}\right)$, 
except for the EoS for EP, which in this simulation was set as an ideal gas with $\gamma=3$. Fig. 7 shows the gas initial geometry.

\subsubsection{Simulation Result}

Figs. 7-9 show the fields of EP concentrations for three moments of time from the simulations 1, 4 and 2, respectively.

Fig. 11 shows the EP bulk concentrations (in the range of 0.001 to 1 ) for the simulation 5. The EP spot is formed in the initial location of the gas and grows with time.

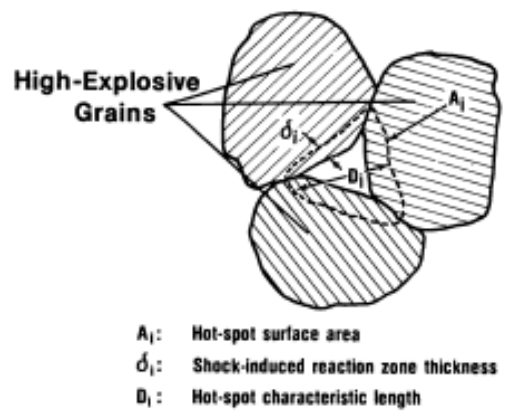

(a)

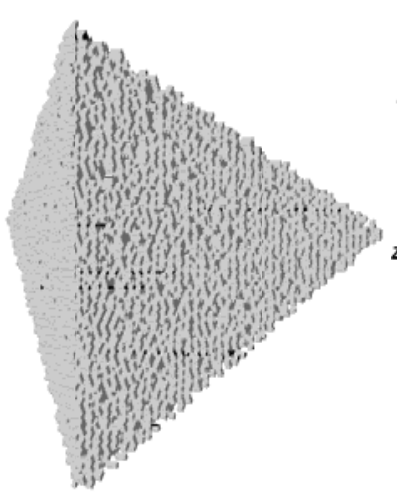

(b)
Fig. 12 shows the time dependences of EP mass in the simulations 1-5. We can see that in the simulation 2, where the kinetics parameter $\mathrm{Z}$ was increased by the order of magnitude, the EP mass is significantly bigger than in other simulations. This simulation was continued only up to $0.42 \mu \mathrm{s}$, since by this time the HE had burnt out nearly in the whole area and further simulation was out of our interest. Here the homogeneous chemical reaction in the HE volume takes place, as well as the acceleration of the spot

Fig. 7 Shape of gas inclusions: (a) experiment [17], (b) computational hot spot and (c) computational full geometry.

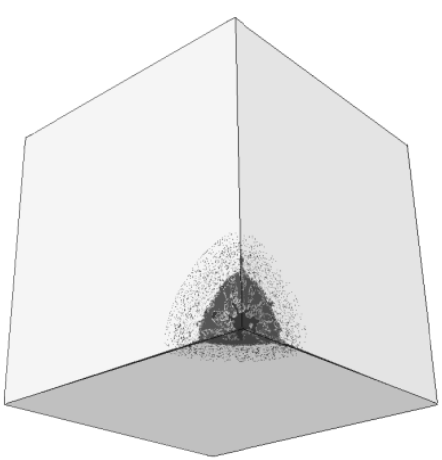

(a)

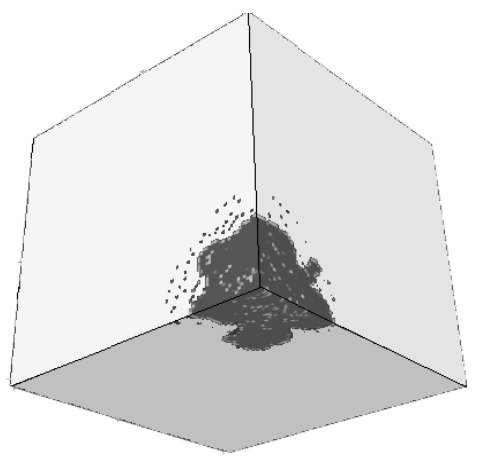

(b)

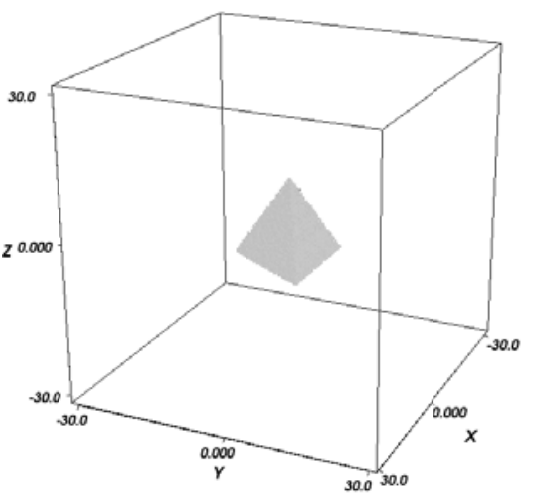

(c)

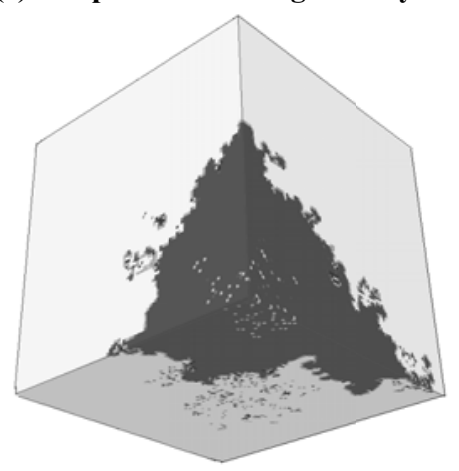

(c)

Fig. 8 Simulation 1. EP concentration (from 0 to 1): (a) $t=1 \times 10^{-8}$, (b) $t=1 \times 10^{-7}$ and (c) $t=1 \times 10^{-6}$.

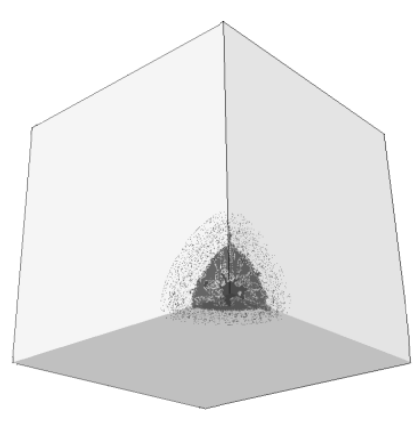

(a)

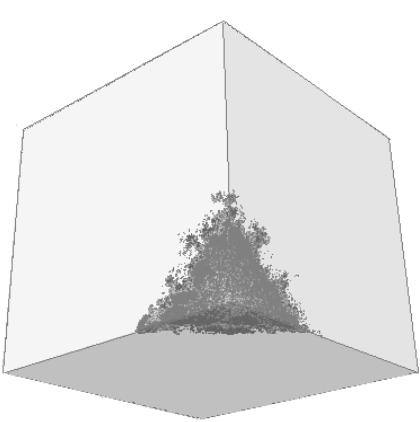

(b)

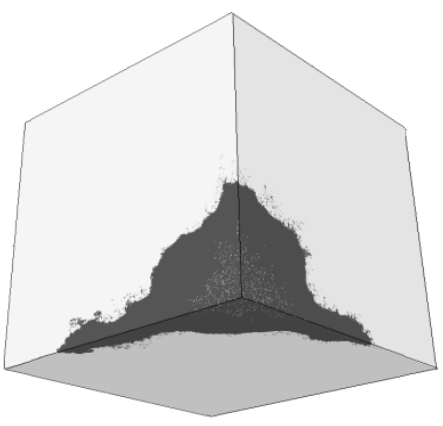

(c)

Fig. 9 Simulation 4. EP concentration (from 0 to 1): (a) $t=1 \times 10^{-8}$, (b) $t=1 \times 10^{-7}$ and (c) $t=1 \times 10^{-6}$. 


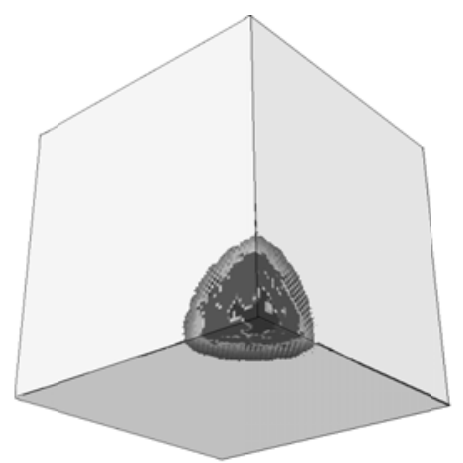

(a)

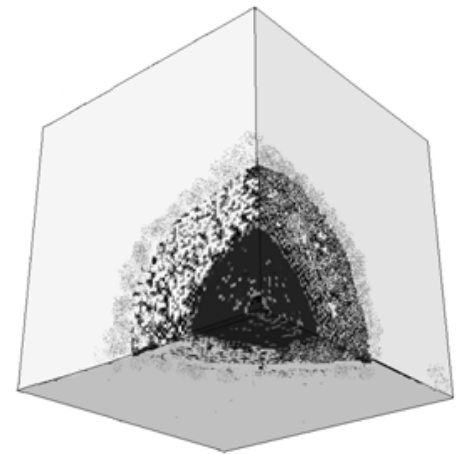

(b)

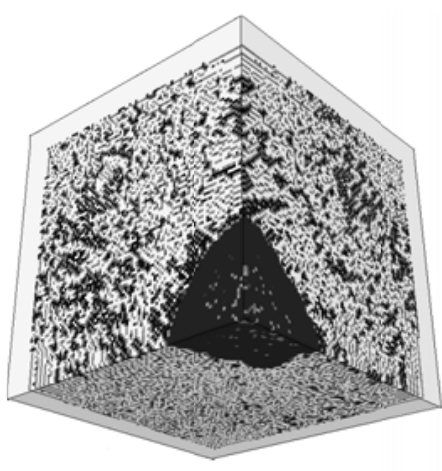

(c)

Fig. 10 Simulation 2. EP concentration (from 0 to 1): (a) $t=1 \times 10^{-8},(b) t=5 \times 10^{-8}$ and (c) $t=1 \times 10^{-7}$.

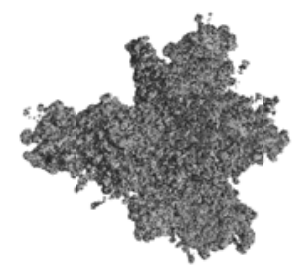

(a)

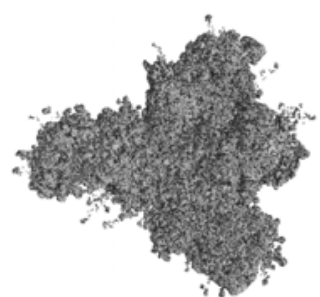

(b)

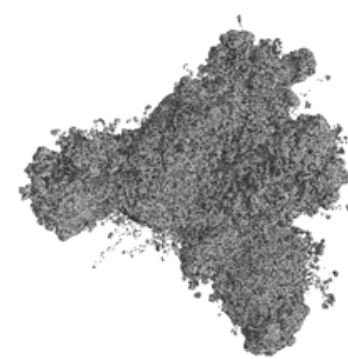

(c)

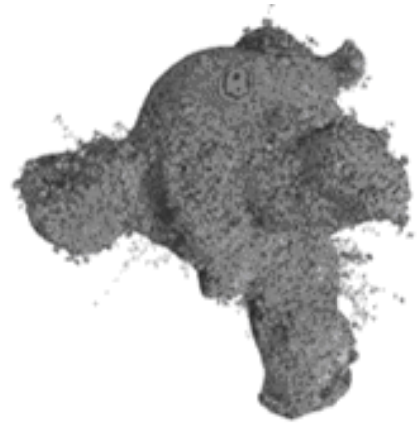

(d)

Fig. 11 Simulation 5. EP concentration (from 0 to 1$)$ : (a) $t=5 \times 10^{-8}$, (b) $t=1 \times 10^{-7}$, (c) $t=2 \times 10^{-7}$ and (d) $t=4 \times 10^{-7}$.

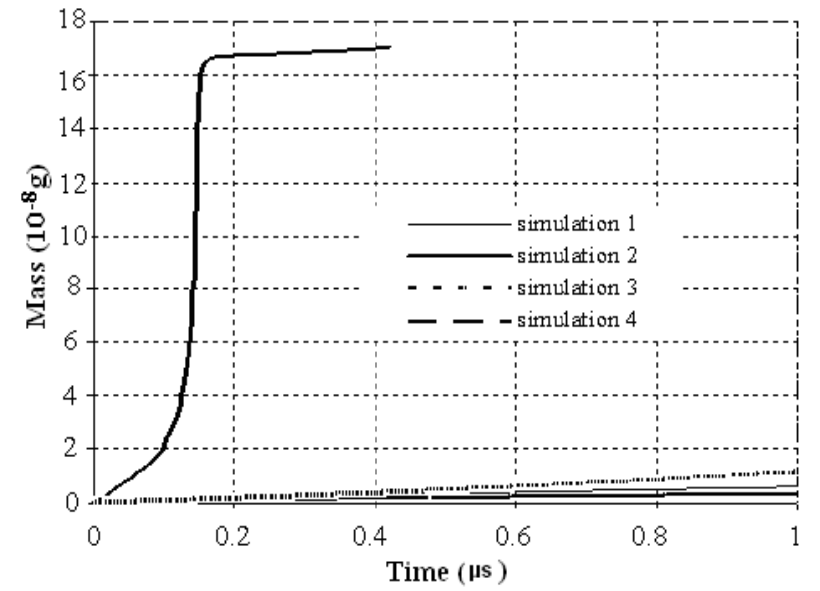

Fig. 12 Time dependences of EP mass in the simulations 1-4.

growth on the background of the homogeneous reaction. This is logical, as the inductance time of homogeneous reactions is very short.

If we intercompare the EP masses from the rest simulations, we will notice that at the growth of the coefficients of thermal conductivity (simulation 3 ) the EP mass grows as well. In the simulation 4 on the similar grid the EP mass is a little lower than in the simulation 1. Two things contribute to this fact. First, the amplitude of the initial perturbations on such grid is smaller, which results in the retarded turbulence development; second, in these simulations rigid walls produce very significant effect, and we can see in the Figs. that the process development is more intense along the walls and weakens as the cell sizes decrease.

\subsection{Determination of the Hot Spot Growth Rate}

Two methods were used for the determination of the hot spot growth rate. The first one over the images. In this case the spot growth rate was about $70 \mathrm{~m} / \mathrm{s}$ in the simulations 1 and 5, which means that in fact, it was the same independently of the setting.

The second criterion is based on the mass of burnt-out HE (mass of resulting EP), modified into spherical spot. In the conditions of developed instability such criterion is the lower evaluation of the spot growth rate. The radius of the sphere after deduction of the gas area is determined under the formula 


$$
\mathrm{R}=\sqrt[3]{\frac{3 \mathrm{~V}}{4 \pi}+\mathrm{R}_{0}^{3}}
$$

where, $\mathrm{V}$ is the EP volume, and $\mathrm{R}_{0}$ - the initial radius of the gas inclusion (for tetrahedron this is the radius of its volume, turned into sphere). Fig. 13 shows the time dependence of the EP spot radius in the simulation 5 obtained using the second criterion. In this simulation the growth rate was about $20 \mathrm{~m} / \mathrm{s}$. Thus, the hypothesis of important role of turbulence in the problem in question has been proved with direct problem simulation; the growth rate of a unit spot increased in the simulations. However, in the same series of simulations the required growth rate was not reached. Therefore we conducted a series of more realistic simulations, which is described in the following section.

\subsection{Modeling of Hot Spot Formation around Gas} Inclusion behind the Wave front Simulation Setting

In this simulation series a more realistic problem was simulated, namely the growth of the spot at the shock wave passage. The problem geometry was a 60 $\times 60 \times 180 \mu \mathrm{m}$ cuboid with two tetrahedron gas inclusions (Fig. 14), which initial dimensions were the same as those in Section 2.1. On the left boundary, on the $\mathrm{x}$-axis, the inflow were specified, which corresponded to the incident shock wave; on the right boundary the outflow condition was set; the rest boundaries were rigid walls. In the simulations the initial parameters for gas and $\mathrm{HE}$, as the shock wave parameters were varied.

Simulation 6: The EoS parameters for gas and EP were the same as in the simulation 5 of Section 2.1, only the HE parameters were different:

$\mathrm{C}_{\mathrm{V}}=0.001 ; \mathrm{C}_{0}=2.9 ; \mathrm{n}=5.4 ; \Gamma=1.25 ; \rho_{00}=1.91$.

A flow with the following parameters was specified on the inflow boundary: $\rho=2.37$; $=8$; $e=0.745$; $\mathrm{u}=0.86$.

Simulation 7: This simulation differed from the simulation 6 by the initial parameters only as follows: for gas $\rho=0.4 ; \mathrm{p}=10^{-4} ; \mathrm{e}=0.00125 ; \mathrm{T}=3.125$; for $\mathrm{HE} \rho=1.91 ; \mathrm{p}=10^{-4} ; \mathrm{e}=0.00004 ; \mathrm{T}=0.04188$; as well as with the inflow $\rho=2.485 ; \mathrm{p}=10$; $\mathrm{e}=0.61$; $\mathrm{u}=1.1$.

Simulation 8: In this simulation the following initial parameters were set:

for gas $\rho=0.001 ; \mathrm{p}=0 ; \mathrm{e}=0.3 ; \mathrm{T}=300$
for $\mathrm{HE} \rho=1.91 ; \mathrm{p}=0 ; \mathrm{e}=0.3 ; \mathrm{T}=300$
inflow $\rho=2.485 ; \mathrm{p}=10 ; \mathrm{e}=0.92 ; \mathrm{u}=1.1$

The quantity $\delta p=(\gamma-1) \rho \varepsilon$ was added to the gas and HE pressure to make the energy and the temperatures balanced at the initial moment, and $\varepsilon=\mathrm{C}_{\mathrm{v}} \mathrm{T}$ was chosen so that the temperature at $\mathrm{t}=0$ was 300 .

\subsubsection{Simulation Results}

Fig. 15 shows the EP bulk concentrations in one of the simulations at several moments of time (the timeline starts at the moment of the SW arrival to the

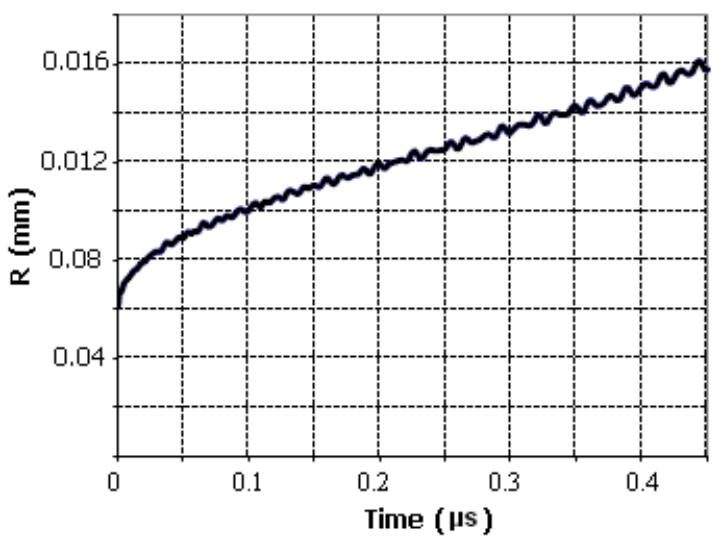

Fig. 13 Time dependence of the EP spot radius in the 3D simulation 5.

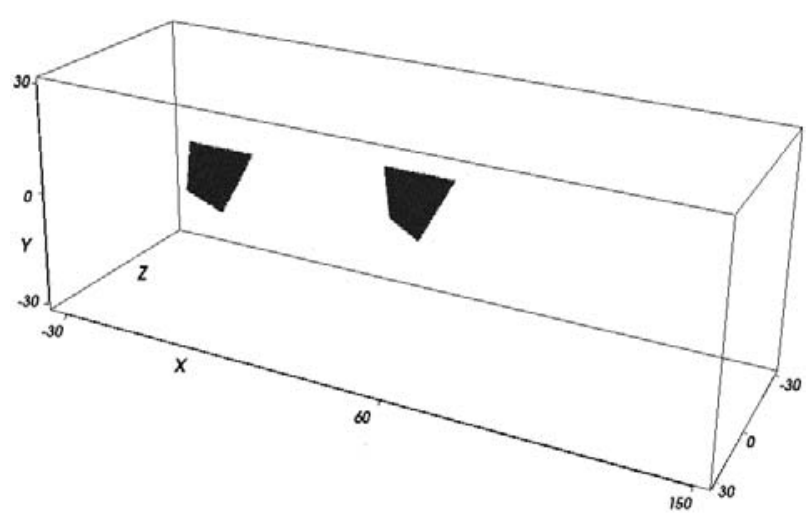

Fig. 14 Initial geometry of 3D simulation with two gas inclusions.

first spot). When the shock wave reached the gas 
inclusions, EP spots were formed there, they moved, grew and then merged. Fig. 16 shows the R-t diagrams of the hot spot sizes in these simulations calculated under the formula 1 without gas inclusion. In all three simulations the time dependence of the spot radius growth reached the approximately linear section, which means that the growth rate was constant. Table 3 shows the growth rates for each spot determined on the linear sections in the simulations. We can see, that the spot growth rates in all the simulations exceeded

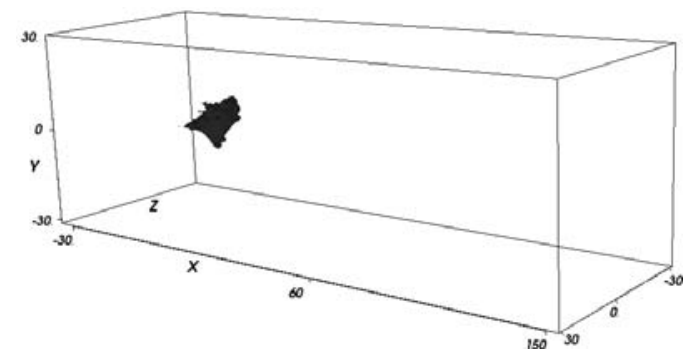

(a)

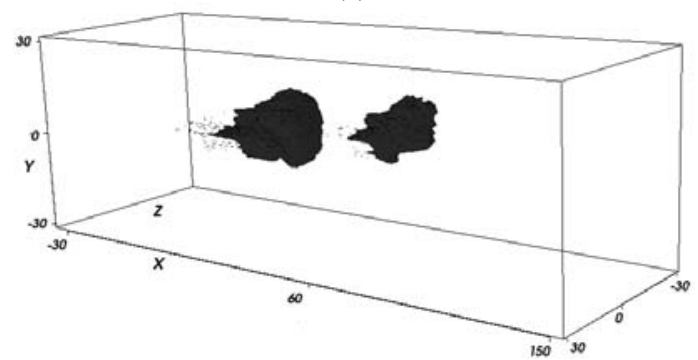

(b)

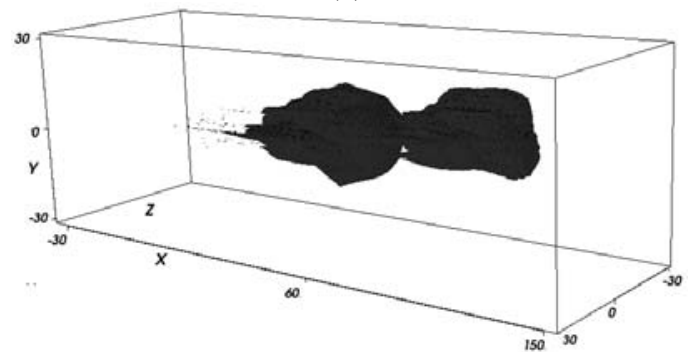

(c)

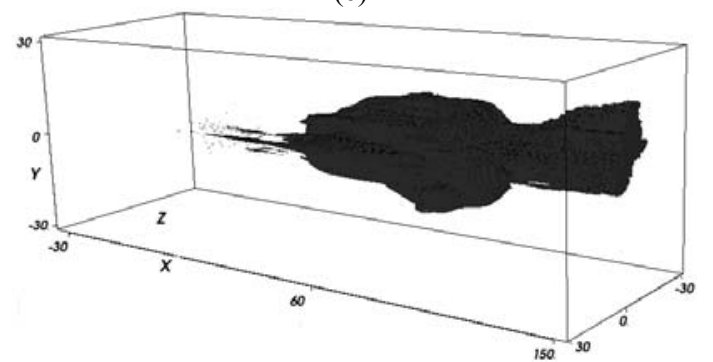

(d)

Fig. 15 EP bulk concentrations in the simulation 8: (a) $t=1 \times$ $10^{-8}$, (b) $\mathrm{t}=3 \times 10^{-8}$, (c) $\mathrm{t}=5 \times 10^{-8}$ and (d) $\mathrm{t}=7 \times 10^{-8}$.

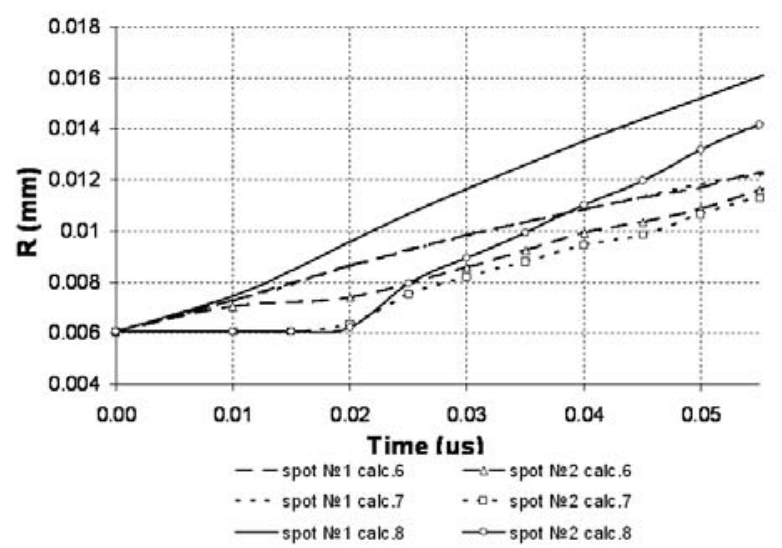

Fig. 16 Time dependences of EP spot radii in the 3D simulations 6-8.

Table 3 Spot growth rates at the linear section $(\mathrm{m} / \mathrm{s})$.

\begin{tabular}{llll}
\hline Simulation & 6 & 7 & 8 \\
\hline Spot 1 & 112 & 122 & 200 \\
Spot 2 & 150 & 154 & 242 \\
\hline
\end{tabular}

$100 \mathrm{~m} / \mathrm{s}$, at that the growth rate of the second spot was significantly larger than that of the first spot. This was due to the fact, that the first spot was under the effect of idealized (not perturbed) shock wave, while the second spot was under the effect of the shock wave perturbed during the first spot passage. This was the additional factor that contributed to the stronger flow turbulence.

\section{Discussion of Results}

At the numerical modeling of the HS burning process with account for turbulent mixing (both $3 \mathrm{D}$ DNS and 2D $\kappa-\varepsilon$ ) and thermal conductivity significantly bigger HS burn rates, as compared with the laminar burning, are obtained. The effect of the rate increase takes place at various problem settings. With the most true-to-life problem settings the HS growth rate was $100 \sim 200 \mathrm{~m} / \mathrm{s}$, which was sufficient for the detonation regime of the HT growth. Thus, the conducted numerical study proves the hypothesis saying that the turbulence in the reaction area is an important factor at the HS burning and it is important to take it into account both in the numerical simulations and the detonation theory.

The physical picture of the HS burning is as follows: 
the shock wave at the compression heats gas inclusions up to a high temperature; when the walls are heated the gas-phase decomposition reaction starts, and the instability develops on the HE/EP interface. The main process of energy transfer goes on turbulently, and, due to the vortex flow, large-scale HE particles mix up and break into smaller-size particles. So, as a result of this event and due to the developed surface of the HE-EP interface the HE has enough time to get heated (energy transfer from EP) and the decomposition reaction effectively continues.

Thus, the turbulent nature of energy transfer and the role of thermal conductivity for the cold HE heating at the developed HE-EP interface and grain refinement are logically substantiated. The experimental studies of the reaction area with high resolution [18, 19] confirm the complicated cellular structure of the detonation front, which perturbation period correlates with the HE grains' sizes.

\section{Conclusions}

The performed numerical modeling has the qualitative research character and does not claim to be absolutely accurate at the evaluation of the process development parameters. Nevertheless, the modeling performed with account for turbulent mixing and thermal conductivity allowed us to obtain the HS growth rates sufficient for reaching the detonation regime of the HS growth. Thus we have acquired the proof of the hypothesis saying that turbulence in the reaction area is an important factor at the HE burning and it should be taken into account in numerical simulations and the detonation theory.

\section{Acknowledgments}

The authors of this paper express their thanks to Glazyrin S. I. for his useful discussions and comments, as well as to Chirkina I. A. for her help in making up the paper.

\section{References}

[1] Andreev, K. K. and Khariton, Yu. B. 1934. "Some
Considerations on the Mechanism of Self-Propagated Reactions." Reports of AS USSR 1:. 402-4.

[2] Belyaev, A. F. 1938. "Initiation of High Explosives Detonation under the Effect of Thermal Impulse." Reports of AS USSR 18: 267-70.

[3] Khariton, Yu. B. and Sukhikh, V. A.. 2003. "Initiation of Flashes in a High Explosive at Short-Time Deformations." In Collection of scientific works.

[4] Bowden, F. P., Stone, M. A. and Tudor, G. K. 1947. "Hot Spots on Rubbing Surfaces and the Detonation of Explosives by Friction.” Proc. R. Soc. A. 188: 329.

[5] Eyring, H., Powel, R. E., Duffrey, G. H. and Darlin, R. B. 1949. "The Stability of Detonation." Chem. Rev. 45: 69.

[6] Bowden, F. P. and Yoffe, A. D. 1952. "Initiation and Growth of Explosions in Liquids Solids." Cambridge, UK: Cambridge University Press.

[7] Tarver, C. M. and Nichols, A. L. 1998. "Hot Spot Growth in a Thermal-Chemical-Reactive Flow Model for Shock Initiation of Solid Explosives." In Proceedings of XI Symposium on Detonation 599-605.

[8] Mazel, P. Saurel, R. Baudin, G. and Loraud, J. C. 1995. "Study of Pyrolysis and Gas Reaction Influence on the Explosive Ignition Mechanism by Viscoplastic Pore Collapse." HDP Congress.

[9] Trofimov, V. S. and Dryomin, A. N. 1966. "On the Substantiation of the Selection Rules for the Detonation Rate." FGV 3: 19-30.

[10] Rybakin, S. S. 1966. "Turbulence at Detonation" FGV 1:29-36.

[11] Morozov, V. G. and Karpenko, I. I. 2002. "Turbulent Mechanism of Energy Transfer at the Hot Spot Growth during Detonation" International conference "Shock Waves in Condensed Matters" 1-6.

[12] Karpenko, I. I. Morozov, V. G. Chernyshova, O. N. and Yanilkin, Yu. V. 2008. "Calculation of the Hot Spot Growth Rates during Detonation with Account for Turbulent Mechanism of Energy Transfer." Chemical Physics 27 (3): 16-20.

[13] Yanilkin, Y. V. Belyaev, S. P. Bondarenko, Y. A. et al. 2008. "Eulerian Numerical Techniques EGAK and TREK for the Modeling of Multidimensional Flows." Science and research edition, RFNC-VNIIEF, 12: 54-65.

[14] Zhernokletov, M. V., Zubarev, V. N. and Telegin, G. S. 1969. "Expansion is Entrops for Explosion Products of Condensed HE." Journal of applied mechanics and technical physics 4: 127-132.

[15] Morozov, V. G., Karpenko, I. I., Kuratov, S. E., Sokolov, S. S., Shamraev, B. N. and Dmitrieva, L. V. 1995. "Theoretical Substantiation of the Phenomenological Model of HE Sensitivity to Shock Waves Basing on TATB." Chemical Physics 14 (2-3): 32.

[16] Morozov, V. G., Karpenko, I. I., Olhov, O. V., Sokolov, 

Turbulent Mechanism of Energy Transfer

S. S., Shamraev, B. N. and Dmitrieva, L. V. et al. 1995. "Computational Modeling with Reference to Experiments on the HE Detonation Initiation and Development Basing on TATB with Account of Desensibilization at the Interaction of Shock was and Detonation Wave." Preprint RFNC-VNIIEF 37.

[17] Tang, P. K. 1988. "Initiation and Detonation of Heterogeneous High Explosive.” A Unified Model,
LASL Report, La-11352-MS.

[18] Plaksin, I. Campos, J. et al. 2005. "Micro-Ejecta from Detonation Front as an Ignored Negative Factor in Performance of Detonation" In Proceeding of 36 International Conference of ICT-Energetic Materials.

[19] Plaksin, I. Campos, J. et al. 2003. "Coarse Explosive Particles of PBX as a Dominant Factor of Detonation Instability." SCCM 887. 